\title{
Video Article \\ Controlling Electrospun Polymer Morphology for Tissue Engineering Demonstrated Using hepG2 Cell Line
}

\author{
Thomas S. R. Bate ${ }^{1}$, Stuart J. Forbes ${ }^{2}$, Anthony Callanan ${ }^{1}$ \\ ${ }^{1}$ Institute for Bioengineering, School of Engineering, University of Edinburgh \\ ${ }^{2}$ Scottish Centre for Regenerative Medicine, University of Edinburgh
}

Correspondence to: Anthony Callanan at anthony.callanan@ed.ac.uk

URL: https://www.jove.com/video/61043

DOI: doi:10.3791/61043

Keywords: Bioengineering, Issue 159, tissue engineering, electrospinning, scaffold morphology, bioengineering, in vitro models, polycaprolactone scaffolds

Date Published: 5/25/2020

Citation: Bate, T.S.R., Forbes, S.J., Callanan, A. Controlling Electrospun Polymer Morphology for Tissue Engineering Demonstrated Using hepG2 Cell Line. J. Vis. Exp. (159), e61043, doi:10.3791/61043 (2020).

\section{Abstract}

Electrospinning affords researchers the opportunity to fabricate reproducible micro to nanoscale polymer fibers. The 3D fibrous architecture of electrospun polymers is regarded as a structural imitation of the extracellular matrix (ECM). Hence, electrospun fibers fabricated from biocompatible polymers have been widely investigated by tissue engineering researchers for their potential role as an artificial ECM for guiding tissue growth both in vitro and in vivo. All cells are acutely sensitive to their mechanical environment. This has been demonstrated by the discovery of multiple mechanotransduction pathways intrinsically linked to the cytoskeletal actin filaments. The cytoskeleton acts as a mechanical sensor that can direct the functionality and differentiation of the host cell depending on the stiffness and morphology of its substrate. Electrospun fibers can be tuned both in terms of fiber size and morphology to easily modulate the mechanical environment within a fibrous polymer scaffold. Here, methods for electrospinning polycaprolactone (PCL) for three distinct morphologies at two different fiber diameters are described. The morphological fiber categories consist of randomly oriented fibers, aligned fibers, and porous cryogenically spun fibers, with $1 \mu \mathrm{m}$ and $5 \mu \mathrm{m}$ diameters. The methods detailed within this study are proposed as a platform for investigating the effect of electrospun fiber architecture on tissue generation. Understanding these effects will allow researchers to optimize the mechanical properties of electrospun fibers and demonstrate the potential of this technology more thoroughly.

\section{Introduction}

Tissue engineering methods have developed over recent decades to provide cells with a biomimetic environment in order to encourage successful tissue regeneration, both for in vitro tissue models and in vivo regenerative techniques. These methods typically encourage cells to form 3D aggregates, either by themselves or with the use of a guiding scaffold ${ }^{1}$. Scaffolds can be fabricated as porous or fibrous polymer nano/ microstructures, or as highly water absorbent hydrogels ${ }^{2,3,4}$. Polymer electrospinning is a common fabrication technique that yields unwoven nano-microscale polymer fiber matrices that can mimic the fibrous architecture of the native extracellular matrix (ECM) ${ }^{5}$. Multiple biocompatible polymers are applicable to the electrospinning process; hence, electrospun fibers are a popular medium to facilitate tissue regeneration ${ }^{6,7,8}$. Electrospun fibers have been utilized in the culture of a range of tissues, demonstrating a promising avenue for inexpensive and reproducible scaffold platforms ${ }^{9,10,11,12,13}$

Cells have an acute sensitivity towards their mechanical surroundings. Previous research has highlighted this important relationship, termed mechanotransduction, which can influence cell function, morphology, and differentiation ${ }^{14,15}$. The basis of the eukaryotic cellular cytoskeleton is constructed from filamentous actin and plays key mechanical roles in cellular division, adhesion, migration, contraction, and morphology. It is manipulated by external mechanical stimuli via connection with extracellular surface proteins ${ }^{16,17,18}$. Morphological influence on stem cell differentiation has been demonstrated in numerous studies by altering the shape of an attachable substrate, which is then able to control cell shape and direct the differentiation process ${ }^{19,20}$. Moreover, the stiffness of the substrate upon which cells attach also influences stem cell fate and lineage ${ }^{21,22}$. Apart from stem cells, these effects have also been observed to affect cells of different lineages ${ }^{23,24}$. Three dimensional tissue engineering methods have shown results akin to those found in 2D mechanotransduction studies with both hydrogels and fibrillar scaffolds ${ }^{25,26,27}$ Further, substrate mechanics are communicated through $3 \mathrm{D}$ cell aggregates via cell-cell signalling ${ }^{28}$. Material mechanics play a crucial role in directing cellular functionality and hence should be a major factor in the design of scaffolds for engineered tissues.

The objective of this method is to understand the effects of electrospun polycaprolactone (PCL) fiber size and morphology on in vitro cell cultures. The method is adapted from previous research focused on kidney tissue engineering ${ }^{9}$. A more detailed step-by-step protocol is offered here in order to facilitate scaffold reconstruction and develop a standardized method that is applicable to all cell types. Six groups of scaffolds were constructed with this method: three are large $5 \mu \mathrm{m}$ fibers (LG) and the other three small $1 \mu \mathrm{m}$ fibers (SM), using different PCL concentrations. Within these LG and SM groups there are three different morphological constructions: random fibers (RA), aligned fibers (AL), and porous cryogenic fibers (CR). These scaffold groups all have inherently different structures, and thus deliver unique local mechanical environments upon which cells attach. To demonstrate the morphological effect this delivers to cells, the hepG2 cell line was seeded onto each group of scaffolds as a representative study. This method is offered as a baseline analysis for electrospun scaffold design for all cell types from stem cells to cell lines and primary cells. 


\section{Protocol}

\section{Solution preparation}

1. Large (random, aligned, and cryogenic) fibers

1. Prepare a $5: 1$ ratio of chloroform and methanol by mixing $50 \mathrm{~mL}$ of chloroform and $10 \mathrm{~mL}$ of methanol in a glass solvent-proof bottle using a glass serological pipette.

CAUTION: Chloroform and methanol are toxic solvents and mixing must be conducted in a fume hood.

2. To create a $19 \mathrm{w} / \mathrm{v} \%$ solution of PCL in 5:1 chloroform:methanol, weigh $1.9 \mathrm{~g}$ of PCL and add to $10 \mathrm{~mL}$ of $5: 1 \mathrm{chloroform}: \mathrm{methanol}$ in a glass solvent-proof vial. Double seal the vial using paraffin film and leave the vial on a roller overnight to allow the PCL to fully dissolve. CAUTION: Mixing must be conducted in a fume hood.

2. Small (random and aligned) fibers

1. To prepare a $7 \mathrm{w} / \mathrm{v} \%$ solution of PCL in hexafluoroisopropanol (HFIP), weigh $0.7 \mathrm{~g}$ of PCL and mix with $10 \mathrm{~mL}$ of $\mathrm{HFIP}$ in a glass solvent-proof vial. Double seal the vial using paraffin film and leave on a roller overnight to allow the PCL to fully dissolve.

CAUTION: HFIP is highly toxic and mixing must be conducted in a fume hood.

3. Small cryogenic fibers

CAUTION: Mixing must be conducted in a fume hood

1. Prepare a $3: 1$ ratio of chloroform and methanol by mixing $30 \mathrm{~mL}$ of chloroform and $10 \mathrm{~mL}$ of methanol in a glass solvent-proof bottle using a glass serological pipette.

2. To create a $14 \mathrm{w} / \mathrm{v} \%$ solution of $\mathrm{PCL}$ in $3: 1$ chloroform:methanol, weigh $1.4 \mathrm{~g}$ of $\mathrm{PCL}$ and add to $10 \mathrm{~mL}$ of $3: 1 \mathrm{chloroform}: \mathrm{methanol}$ in a glass solvent-proof vial. Double seal the vial using paraffin film and leave on a roller overnight to allow the PCL to fully dissolve.

\section{Electrospinning setup}

NOTE: The parameters must be set before beginning the electrospinning process.

1. Needle size and placement

1. For large fibers (random, aligned, and cryogenic) use a $1 \mathrm{~mm}$ OD/0.8 mm ID brass needle and place the needle $230 \mathrm{~mm}$ away from the mandrel surface.

2. For small fibers use a $1 \mathrm{~mm}$ OD/0.4 $\mathrm{mm}$ ID brass needle and place the needle $15 \mathrm{~mm}$ away from the mandrel surface.

2. Lateral needle motion

NOTE: The scaffolds detailed in Figure 1 were fabricated with a $100 \mathrm{~mm}$ needle traverse at $50 \mathrm{~mm} / \mathrm{s}$, with repeated motion left and right throughout the process.

1. To set the lateral motion, press Motion | TNS 1 and set Start position [mm] to $-\mathbf{5 0}$, Distance [mm] to 100, Speed [mm/s] to $\mathbf{5 0}$ and Turn Delay [ms] to 750 .

3. Syringe pump flow rate

1. For large fibers (random, aligned, and cryogenic), press the Material tab on the user interface and set the syringe pump to $4 \mathrm{~mL} / \mathrm{h}$.

2. For small (random and aligned) fibers, set the syringe pump to $0.8 \mathrm{~mL} / \mathrm{h}$.

3. For small cryogenic fibers, set the syringe pump to $2.5 \mathrm{~mL} / \mathrm{h}$.

4. Voltage settings

1. For the large fibers, set the needle voltage at $+15 \mathrm{kV}$ and the mandrel voltage at $-4 \mathrm{kV}$ by pressing the Voltage tab on the user interface and changing the positive and negative voltage values.

2. For the small (random and aligned) fibers, set the needle voltage at $+17 \mathrm{kV}$ and the mandrel voltage at $-4 \mathrm{kV}$.

3. For the small cryogenic fibers set the needle voltage at $+18 \mathrm{kV}$ and the mandrel voltage at $-4 \mathrm{kV}$.

5. Mandrel rotation (large and small fibers)

1. For random fibers, press Motion | Target and set the mandrel rotation speed to $250 \mathrm{rpm}$.

2. For aligned fibers, set the mandrel rotation speed to $1,800 \mathrm{rpm}$.

3. For cryogenic fibers, set the mandrel rotation speed to $180 \mathrm{rpm}$.

\section{Electrospinning of random and aligned fibers (large and small fibers)}

1. Insert the needle (refer to section 2.1 for information on needle size and placement) into $1 \mathrm{~mm}$ bore PTFE tubing and secure within the electrospinner needle holder.

2. Under a fume hood, transfer the $10 \mathrm{~mL}$ of polymer solution into a polypropylene or glass (solvent-proof) syringe. Make sure to remove any bubbles by letting the syringe stand before priming.

3. Connect the syringe to the needle via the PTFE tubing and secure the syringe within the syringe pump. Once connected, prime the PTFE tubing by squeezing the syringe plunger and filling the tube with polymer solution.

4. Wrap the mandrel in aluminum foil and secure onto the mandrel rotation device.

5. Switch on the mandrel rotation by pressing Motion | Target | Rotate and the syringe pump by pressing Material | Start Pump 1. 
6. When a small bead of polymer has formed at the end of the needle, switch on the voltage by pressing ACTIVATE HIGH VOLTAGE. A jet of polymer should then be emitted from a conical protrusion of polymer solution, called the Taylor cone. Check the mandrel to confirm fiber deposition on the mandrel surface.

7. Run the electrospinning process until the desired amount of PCL solution is electrospun. NOTE: For the scaffolds presented in the Figure 1, $4 \mathrm{~mL}$ of solution was electrospun.

8. Once the electrospinning has terminated, remove the aluminum foil covered with the fiber from the mandrel and leave in a fume cupboard overnight to allow for excess solvent evaporation.

9. After evaporation, store the fibers at room temperature (RT).

\section{Electrospinning of cryogenic fibers}

1. For cryogenic electrospinning, use the cryo-spinning mandrel. Repeat steps 3.1-3.3 then proceed with the following steps.

2. Wrap the mandrel in aluminum foil.

3. Fill the mandrel with dry ice pellets. Secure the cryo-spinning mandrel cover onto the mandrel to ensure that dry ice cannot escape the mandrel but pressure can escape. Ice crystals should form on the surface of the mandrel almost immediately. CAUTION: Use protective gloves when handling dry ice to avoid cold burns.

4. Secure the mandrel onto the mandrel rotation device and immediately switch on the mandrel rotation by pressing Motion | Target | Rotate and the syringe pump by pressing Material | Start Pump 1.

5. Once a bead of polymer has formed at the end of the needle, switch on the voltage by pressing ACTIVATE HIGH VOLTAGE. A jet of polymer should then be emitted from a conical protrusion of polymer solution (i.e., the Taylor cone). Check the mandrel to confirm fiber deposition on the mandrel surface.

6. After $1 \mathrm{~h}$ of electrospinning, pause the process by pressing STOP ALL and top up the mandrel with dry ice. Ensure adequate protection from solvent vapor while refilling the mandrel. Perform this for a total of $3 \mathrm{x}$ for $4 \mathrm{~h}$ total of electrospinning time.

NOTE: Electrospinning can be resumed by repeating steps 4.4 and 4.5 .

7. Once the electrospinning ends, carefully remove the mandrel and immediately place it into a freeze drier for $24 \mathrm{~h}$ to sublimate the ice crystals on the mandrel surface.

8. After sublimation remove the fiber-covered foil from the mandrel and store at RT, taking care to avoid crushing.

\section{Scaffold punching}

1. Punch scaffolds from the sheet of fibers using a 10-12 $\mathrm{mm}$ biopsy punch. If the fibers remain adhered to the aluminum foil, place the scaffolds in $70 \%$ ethanol to facilitate detachment.

\section{Sterilization and plasma coating}

1. Place the scaffolds ( 1 scaffold per well) into a 24 or 48 well plate and wash them $3 x$ in $70 \%$ ethanol. Leave submerged in the final wash, place into a $-80^{\circ} \mathrm{C}$ freezer, and allow to freeze.

2. Once frozen, place the plate into a freeze drier and lyophilize for $24 \mathrm{~h}$. The dried scaffolds can now be plasma coated. NOTE: If there is no access to a plasma coater, submerge the scaffolds in $1 \%$ antibiotic-antimycotic (Anti-Anti) in phosphate buffered saline (PBS) and leave to incubate at $37^{\circ} \mathrm{C}$ overnight.

3. Place the plate into the plasma chamber and remove the lid. Run the plasma coater at 500 mTorr and medium radiofrequency (RF) power for $30 \mathrm{~s}$.

4. After plasma coating, submerge the scaffolds in $1 \mathrm{~mL}$ of PBS and $1 \%$ Anti-Anti. The scaffolds should readily absorb the PBS solution. NOTE: The scaffolds are now ready to be seeded with cells.

\section{Preparation and seeding of cells}

NOTE: The basic cell culture technique provided below is for the hepg2 cells used for demonstration purposes in the representative results. These principles can be applied to any adherent cell type; however, different cells may require different materials and different processes. Cell handling must be conducted in a biological safety cabinet, following aseptic technique.

1. Take $1 \times 10^{6}$ cryopreserved hepg2 cells and seed into a T75 cell culture flask with $12 \mathrm{~mL}$ of complete media consisting of Eagle's minimum essential media (EMEM) supplemented with $10 \%$ fetal bovine serum, $100 \mathrm{U} / \mathrm{mL}$ penicillin-streptomycin, $5 \mathrm{mM} \mathrm{L-glutamine,} \mathrm{and} 1 \%$ nonessential amino acids.

2. Once the cells are at $80 \%$ confluence detach the cells from the culture flask by washing $3 x$ in PBS and incubating in $2 \mathrm{~mL}$ of trypsin at $37{ }^{\circ} \mathrm{C}$ for 4 min. After tapping the flask, the cells should detach.

3. Deactivate the trypsin by adding $8 \mathrm{~mL}$ of complete media. Transfer the cell suspension into a $15 \mathrm{~mL}$ tube and spin down the cells in a centrifuge at $120 \times g$ for $5 \mathrm{~min}$.

4. Remove the supernatant above the cell pellet with an aspirating pipette and replace with $5 \mathrm{~mL}$ of complete media. Resuspend the cells by pipetting up and down with a $5 \mathrm{~mL}$ serological pipette.

5. Count the resulting cell suspension via the trypan blue exclusion method. Take $100 \mu \mathrm{L}$ of cell suspension and add to $100 \mu \mathrm{L}$ of trypan blue in a $1.5 \mathrm{~mL}$ vial. Introduce the mixture onto a hemocytometer under a glass coverslip and count the cells under a microscope.

6. Adjust the cell concentration to $2.6 \times 10^{6} \mathrm{cells} / \mathrm{mL}$ by adding or removing complete media. To remove media, centrifuge cells at $220 \times g$ for 5 min, remove the required media, and resuspend the cells.

7. Aspirate the $1 \%$ Anti-Anti in PBS from the well plate and seed $50 \mu \mathrm{L}$ of $2.6 \times 10^{6}$ cells $/ \mathrm{mL}$ cell suspension onto the scaffold surface using a $200 \mu \mathrm{L}$ pipette. 
8. Allow cells to attach under incubation at $37^{\circ} \mathrm{C}$ and $5 \% \mathrm{CO}_{2}$ for $1-3 \mathrm{~h}$, making sure they do not dry out. Once attached, add $300 \mu \mathrm{L}$ (for a 48 well plate) or $500 \mu \mathrm{L}$ (for a 24 well plate) of culture media and incubate at $37^{\circ} \mathrm{C}$ and $5 \% \mathrm{CO}_{2}$.

NOTE: Standard cell culture techniques can be used from here until further functional analysis.

\section{Representative Results}

Figure 1 shows scanning electron microscope (SEM) images of both small $(\sim 1 \mu \mathrm{m})$ and large fibers $(\sim 5 \mu \mathrm{m})$ fabricated from PCL solutions. The fibers were sputter-coated with gold-palladium (60:40) for $30 \mathrm{~s}$ using a sputter coater prior to SEM imaging. Large fibers were produced using a high $19 \mathrm{w} / \mathrm{v} \%$ concentration of PCL in a 5:1 solution of chloroform and methanol to create a high viscosity PCL solution. This high viscosity solution was then electrospun at a high flow rate to emit a large thread of polymer solution within the electric field. Therefore, a large $230 \mathrm{~mm}$ distance between the needle and the mandrel was necessary to ensure effective evaporation of the solvent before deposition on the mandrel. Conversely, smaller fibers were produced using a low $7 \mathrm{w} / \mathrm{v} \%$ concentration of PCL in HFIP to create a low viscosity PCL solution. Electrospinning the low viscosity PCL solution at a low flow rate ensured that a thinner thread of PCL solution was emitted from the needle within the electric field. The thinner thread facilitated a quicker evaporation of solvent and the needle needed to be closer to the mandrel at $70 \mathrm{~mm}$ to ensure a stable deposition of fibers. For electrospinning small cryogenic fibers, altering the PCL solution was necessary to achieve a reliable protocol. PCL in HFIP electrospun onto the cryogenic mandrel repeatedly resulted in a disintegrated fiber structure. Electrospinning a $14 \mathrm{w} / \mathrm{v} \%$ PCL concentration in 3:1 chloroform methanol solved this problem; however, this created a larger variation in fiber size throughout the scaffold. The extent of fiber alignment (fiber radius and orientation) for each scaffold was analyzed through the Image J DiameterJ plugin ${ }^{29}$ and shown in Figure 2 and Figure 3.

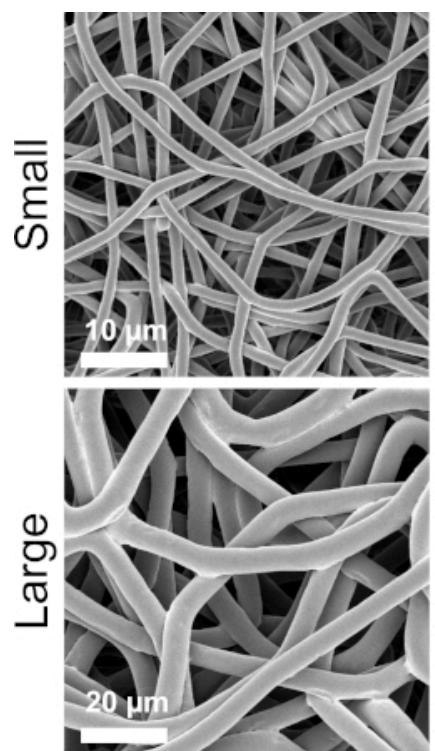

Random

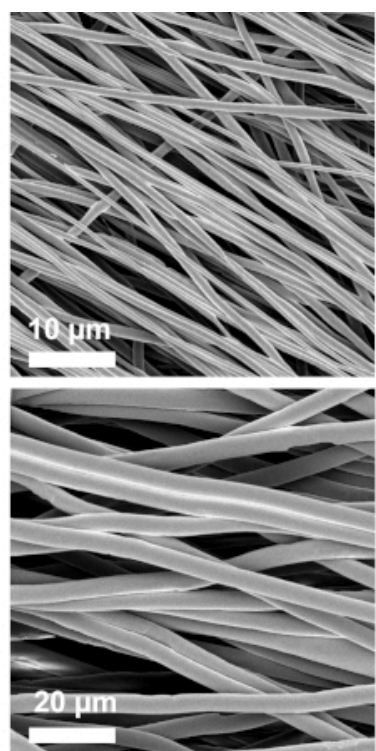

Aligned
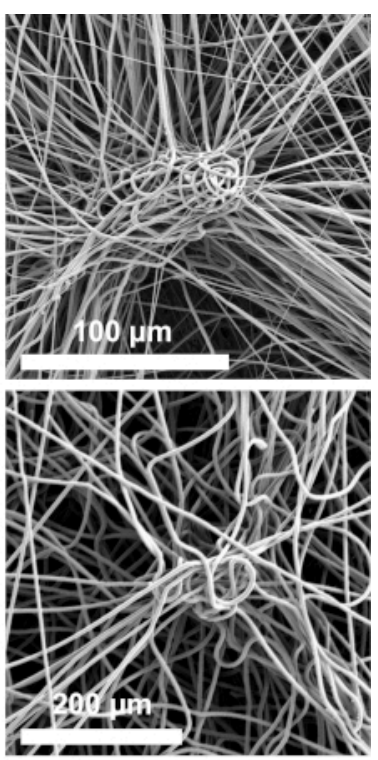

Cryogenic

Figure 1: SEM images showing the morphological qualities of each scaffold group. The top row shows the small fibers and the bottom row the large fibers. Morphological groups are listed left to right as random fibers, aligned fibers, and cryogenic fibers, respectively. Please click here to view a larger version of this figure. 
Fibre radius
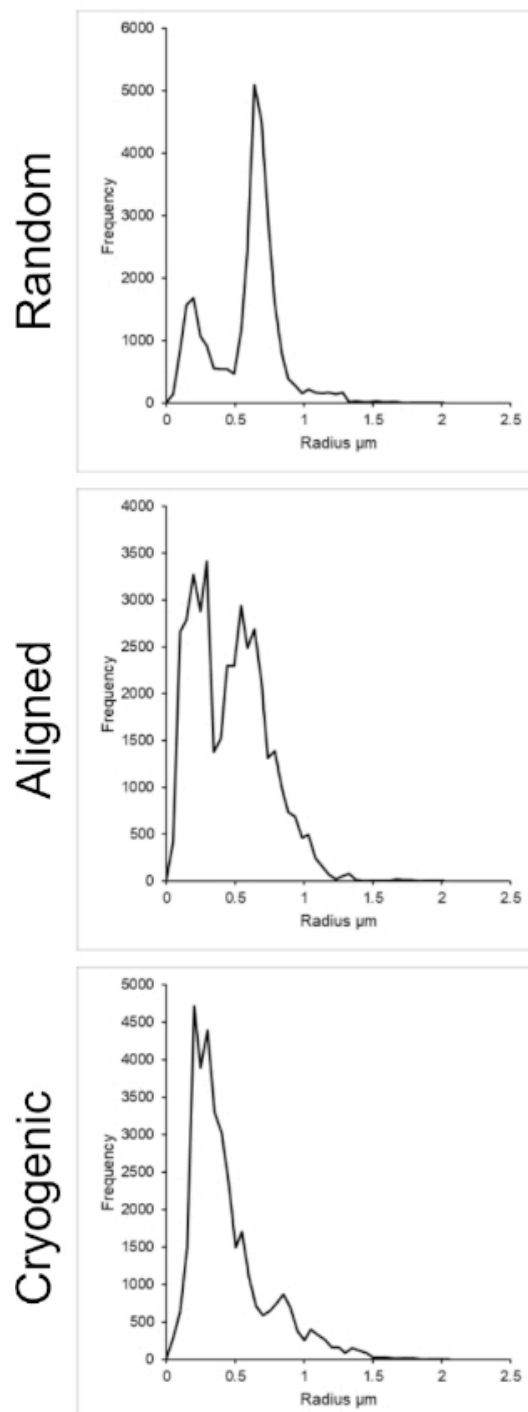

\section{Fibre orientation}
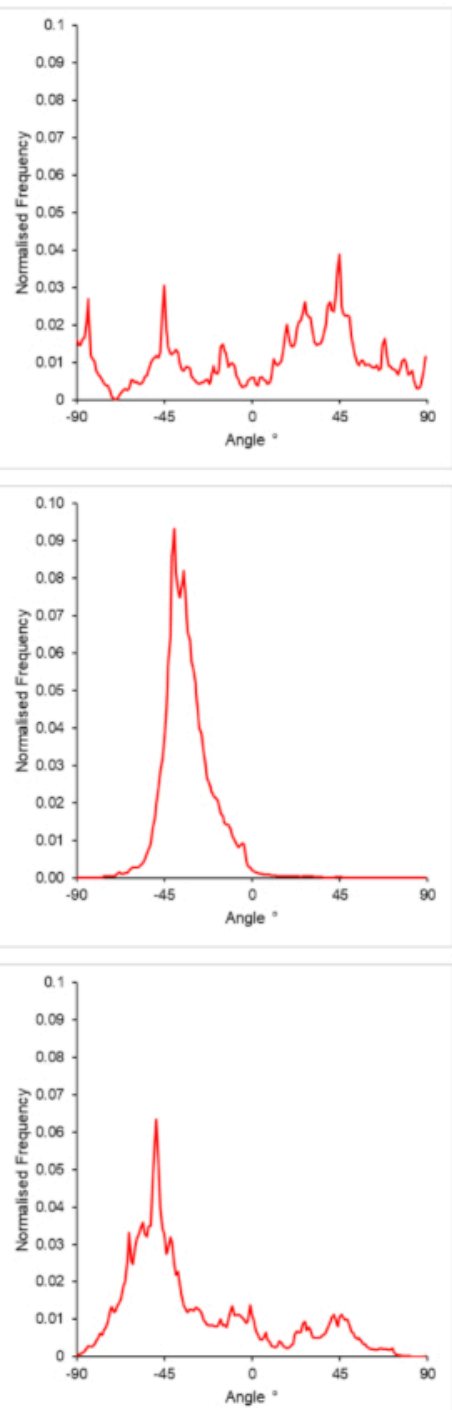

Figure 2: Fiber radius (left) and fiber orientation (right) data from the DiameterJ plugin for the small set of fibers. Morphology listed from top to bottom as random fibers, aligned fibers, and cryogenic fibers, respectively. Please click here to view a larger version of this figure. 
Fibre radius
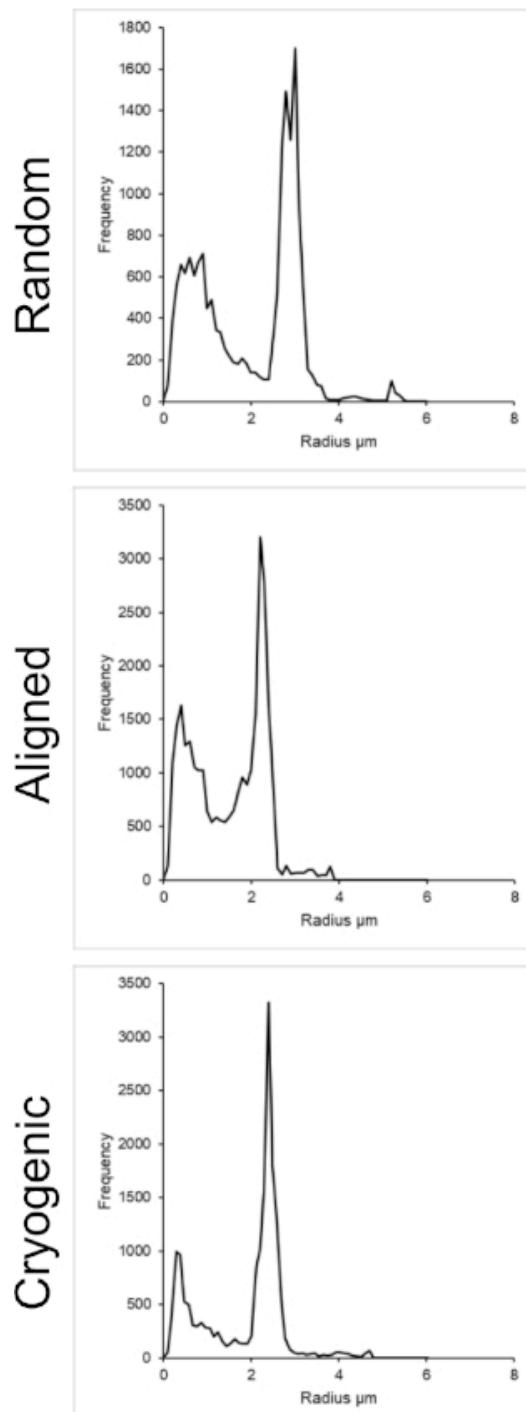

Fibre orientation
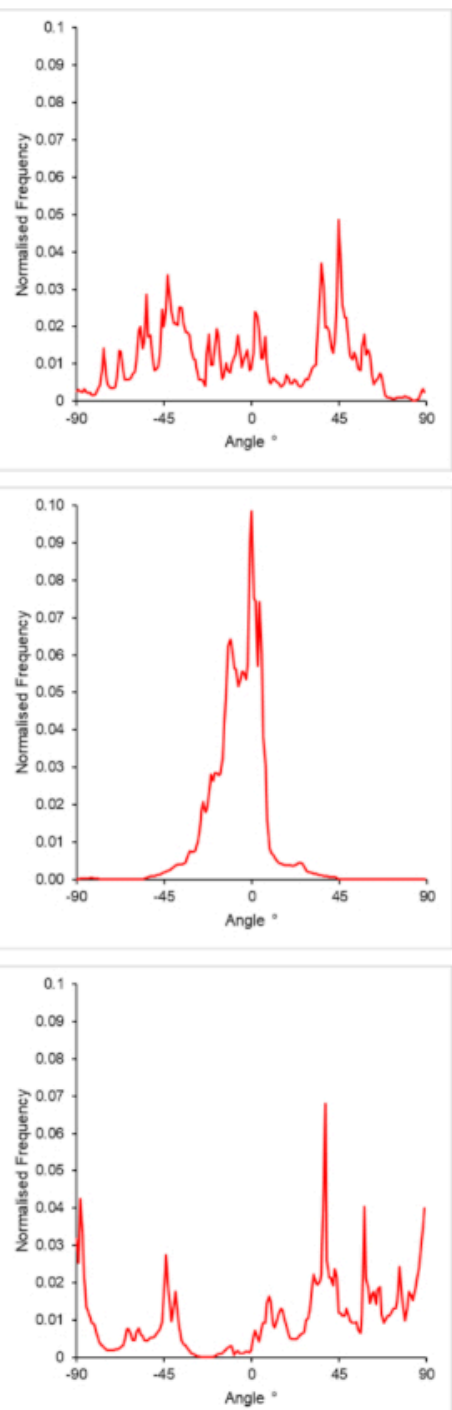

Figure 3: Fiber radius (left) and fiber orientation (right) data from the DiameterJ plugin for the large set of fibers. Morphology listed from top to bottom as random fibers, aligned fibers, and cryogenic fibers, respectively. Please click here to view a larger version of this figure.

This method offered us a set of scaffolds with six distinctly different tensile properties as determined using a tensile testing machine according to previously described methods ${ }^{9}$. As shown in Table 1 the macroscale Youngs' Moduli ranges from $46.94 \pm 3.48 \mathrm{MPa}$ in the large aligned fibers to $0.20 \pm 0.01 \mathrm{MPa}$ in the large cryogenic fibers. Physiological tissue stiffness values range from $100 \mathrm{~Pa}$ (neural tissue) to $2-4 \mathrm{GPa}$ (bone tissue) ${ }^{30}$; all of the scaffold stiffness values reported here were within this range.

\begin{tabular}{|l|l|l|l|l|l|}
\hline \multirow{2}{*}{ Scaffold } & Youngs' modulus $(\mathrm{MPa})$ for strain\% & \multicolumn{3}{l|}{} \\
\cline { 2 - 6 } & $0-2 \%$ & $2-4 \%$ & $4-6 \%$ & $6-8 \%$ & $8-10 \%$ \\
\hline SM.RA & $11.14 \pm 2.13$ & $9.31 \pm 0.43$ & $6.75 \pm 0.64$ & $4.75 \pm 0.59$ & $3.32 \pm 0.40$ \\
\hline SM.AL & $27.94 \pm 8.63$ & $28.27 \pm 7.36$ & $16.34 \pm 4.87$ & $9.10 \pm 3.75$ & $5.85 \pm 3.11$ \\
\hline SM.CR & $0.27 \pm 0.11$ & $0.31 \pm 0.44$ & $0.31 \pm 0.65$ & $0.29 \pm 0.59$ & $0.26 \pm 0.40$ \\
\hline LG.RA & $9.23 \pm 0.78$ & $8.22 \pm 0.87$ & $5.96 \pm 0.68$ & $3.96 \pm 0.59$ & $2.63 \pm 0.45$ \\
\hline LG.AL & $46.94 \pm 3.48$ & $38.03 \pm 2.46$ & $27.69 \pm 1.22$ & $16.10 \pm 1.61$ & $7.87 \pm 1.32$ \\
\hline LG.CR & $0.20 \pm 0.01$ & $0.16 \pm 0.01$ & $0.12 \pm 0.01$ & $0.10 \pm 0.01$ & $0.09 \pm 0.01$ \\
\hline
\end{tabular}

Table 1: Tensile testing data for each scaffold group at intervals of $2 \%$ strain. SM.RA = Small random, SM.AL $=S$ mall aligned, SM.CR = Small cryogenic, LG.RA = Large random, LG.AL = Large aligned, and LG.CR = Large cryogenic. Data presented as mean $\pm S D, n=5$. 
Figure 4 shows the DAPI and phalloidin staining used to visualize the cellular morphology. The staining method used is established in the literature ${ }^{11,12}$. Images were obtained using a microscope (Table of Materials) with a 40x magnification objective. The images show that the aligned fibers produced by this method elongated the hepG2 cell morphology and directed proliferating cells along the length of the fibers, creating directionality within the cell culture. In contrast, the hepG2 cells on random fibers did not display the directional proliferation and elongation observed in the aligned structures. On the random structures the hepG2 cells displayed a more regular morphology with extended protrusions attaching to the surrounding PCL fibers.

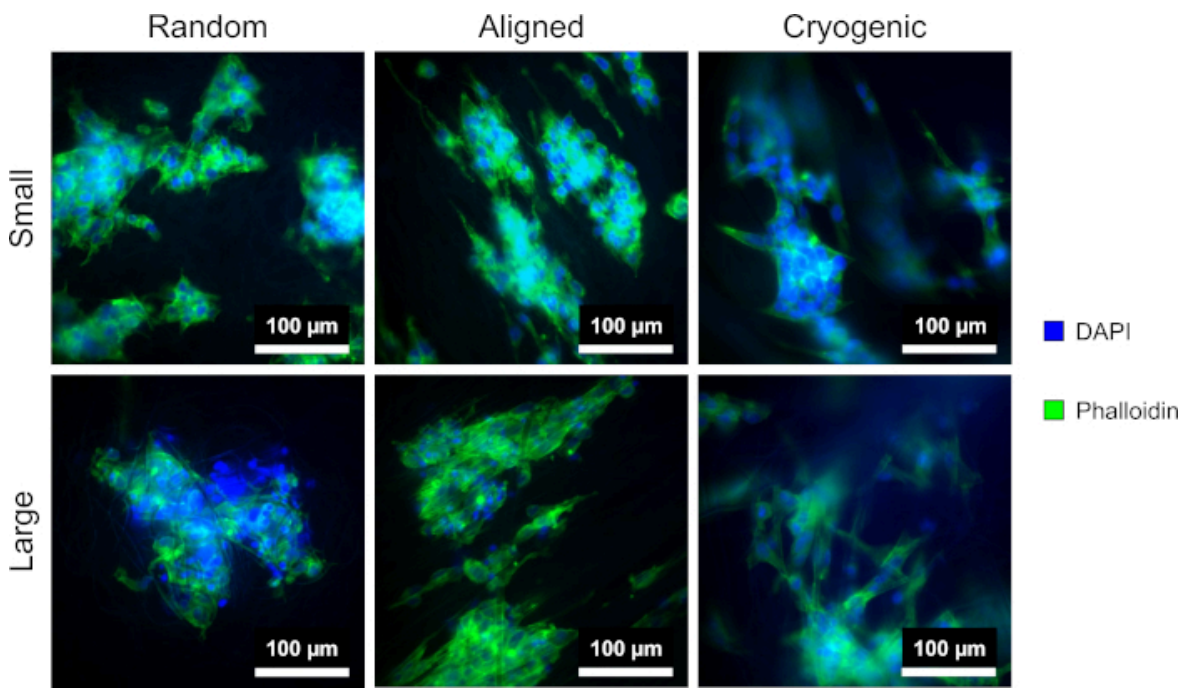

Figure 4: DAPI (blue) and phalloidin (green) stained hepG2 cells on the different scaffold morphologies: random (left), aligned (middle), and cryogenic (right). The top row shows small fibers and the bottom row shows large fibers. Please click here to view a larger version of this figure.

\section{Discussion}

The creation of different fiber sizes was achieved using a combination of different solvents, PCL concentrations, and electrospinning parameters. The type of polymer, its molecular weight, and the solvent strongly affect the viscosity and charge properties of the resulting solution and therefore bear a strong influence on the electrospinning properties ${ }^{31}$. The voltages displayed in this method are subject to change based upon the conditions in which the electrospinning is conducted. Temperature and humidity affect the behavior of the electrospinning process ${ }^{32}$. Therefore, the reader is advised to be prepared to change the voltage to counter any hindrances and achieve a stable Taylor cone. It is recommended to house the electrospinner within an environmental control enclosure to reduce batch-to-batch variability in the electrospinning process.

Achieving the different morphologies presented in this method is possible due to the capabilities of the IME electrospinning device. A variable speed mandrel is essential for controlling fiber alignment. Mandrel rotation at a low rpm (<250 rpm) yields randomly oriented fibers due to the behavior of the polymer jet. When the charged polymer solution is emitted from the needle, the internal charges and aerodynamic forces induce a chaotic whipping effect that is utilized to lay down a chaotic arrangement of fibers on the mandrel ${ }^{33,34}$. This whipping effect can be overcome by increasing the mandrel rotation speed above 1,800 rpm. Fibers deposited on such a high rpm mandrel exhibit an aligned structure because the surface speed is enough to effectively counter any transverse movement exhibited by the whipping fiber. It is important to note that when high mandrel speeds are used, it is likely to disrupt the surrounding air, which can have an adverse effect on the production of a stable Taylor cone.

Highly porous cryogenic scaffolds are produced by filling the mandrel with dry ice at $-78.5^{\circ} \mathrm{C}$. This reduces the temperature of the mandrel and promotes condensation and freezing of water droplets on the surface. The crystals formed exhibit peaks that emerge from the mandrel surface and the fibers are deposited throughout the peaks. Once the ice crystals are sublimated out, a porous structure of fibers remains on the mandrel ${ }^{35,36}$. The weight and fragility of the crystals limit the ability to create porous aligned fibers, as too high mandrel speeds (>250 rpm) result in the crystals and fibers detaching from the mandrel due to excessive centripetal and aerodynamic forces. It is possible to face difficulties with this method due to the fragility of the structures formed. Care must be taken when handling the mandrel and also when handling the scaffolds afterwards, because the structures are prone to being flattened when squeezed and folded when removing from liquids. There is also the possibility that solutions that electrospin successfully without the cryogenic mandrel can be fatally disrupted by the temperature change and the crystal structures. When cryo-spinning, the amount of ice formed on the mandrel surface is highly dependent on the humidity, producing variability in the final result. It is therefore highly recommended to contain the electrospinning process within an environmental control enclosure.

To assess cellular performance on these scaffolds, it is necessary to sterilize the material beforehand. There are many options available for sterilizing polymers, including chemical methods, radiation methods, and heat-based methods ${ }^{37,38}$. It is important to assess the effectiveness of each method and the suitability for both the use of the material and for the material itself. This protocol uses sterilization in $70 \%$ ethanol before lyophilizing, plasma treating, and submerging in 1\% Anti-Anti solution. The use of ethanol allows proteins and microorganisms to be denatured and dehydrated while not disrupting the PCL material ${ }^{37}$. The use of stronger solvents may result in the dissolution of the PCL. Plasma coating was incorporated in this method primarily to increase the hydrophilicity of PCL, which is notoriously hydrophobic, and improve cell attachment ${ }^{39,40}$. Conveniently, this also works as a secondary sterilization step, but care must be taken to keep the scaffolds in a sterile environment pre and post plasma treatment. This method has proven suitable for in vitro studies with antibiotic treated media. However, for in 
vivo applications, more robust options such as ethylene oxide $(\mathrm{EtO})$ and gamma exposure must be considered. Once sterilization has been performed, cells can be seeded onto the scaffold and standard cell culture techniques can be applied to form 3D cell cultures in vitro.

It is important to note that the stiffness values should be attributed to the different microstructures formed within the scaffolds. The bulk material $(P C L)$ remains constant between scaffolds. Therefore, the bulk material stiffness does not change between groups. The cryogenic scaffolds display a much lower stiffness than the other groups due to the reduced connectivity within the fiber matrix, which can be seen in Table 1 . The reduced connectivity is thought to induce a more bending-dominant deformation as opposed to stretching-dominant deformation within the fibrous structure ${ }^{41}$. Interestingly, the scaffold stiffness is not highly dependent on fiber size in the random and cryogenic groups. However, the longitudinal stiffness of the aligned fibers shows a significantly higher dependence on fiber size with the Youngs' Modulus at $0-2 \%$ strain being $27.94 \pm 8.63 \mathrm{MPa}$ for small fibers and $46.94 \pm 3.48 \mathrm{MPa}$ for large fibers. Due to the stretching nature of the tensile test, this implies a higher bulk $\mathrm{PCL}$ density within the longitudinal cross section. It is necessary to reiterate that the mechanical data presented here is a macroscale mechanical snapshot of the fibrous architectures. Further micromechanical characterization would be beneficial to fully understand the mechanical influences on the cell scale.

Both random and aligned architectures were included in this method to provide a comparison between isotropic and anisotropic morphologies. The extent of fiber alignment can be observed in the SEM images in Figure 2 and the fiber analyses presented in Figure 2 and Figure 3. Anisotropic properties are observed throughout many of the tissues in the body. In particular, this is commonly observed in aligned cellular structures such as those found in muscle and nerve tissues. Aligned polymeric fiber structures offer the capability of recapitulating these aligned structures in vitro ${ }^{42,43}$. As described before, cell function is sensitive to morphological and mechanical changes, so further functional analysis should be conducted on scaffold-bound cells to determine the biological influence of each scaffold type.

Compared to other existing scaffold fabrication techniques, this method provides a simple way to produce microscale scaffold structures with comparatively high control over the mechanical properties and morphology. Alternative PCL scaffold fabrication such as phase separation, salt leaching, and gas foaming allow for morphological control in terms of void space and pore size. However, the pore and structural geometry remains largely the same ${ }^{44,45,46,47}$. Therefore, qualities such as the level of isotropy cannot be altered as easily in comparison to electrospinning. Hydrogel materials, popular for scaffold production, provide the means to alter the stiffness of the polymer substrate through alteration of the level of cross-linked polymer chains ${ }^{48}$. It is also possible to 3D print via a variety of methods, providing excellent control over morphology ${ }^{49}$. However, achieving electrospinning scale resolution in biocompatible hydrogel materials remains a challenge to be practically implemented ${ }^{50,51}$ Electrospun scaffolds have featured commonly in tissue engineering research throughout the last decade, and the introduction of new materials and applications with different cell types is always being explored. While new materials are constantly under development for the purpose of electrospinning scaffolds, opportunities for further biological characterization of existing electrospinning materials and methods remain. The method described is proposed as a method for facilitating in vitro biological study, as it is directly applicable to basic cell culture techniques.

\section{Acknowledgments}

This work was supported by the Engineering and Physical Sciences Research Council Grant Ref: EP/N509644/1, the UKRMPII Grant Ref: MR/ L022974/1 and the MRC CCBN Grant Ref: MR/L012766/1. The authors would like to acknowledge Dr. Alison McDonald and Dr. David Kelly (COIL) for assisting with and facilitating the microscopy imaging and Prof. Alistair Elfick for access to the laboratory facilities (Institute for Bioengineering).

\section{References}

1. Akter, F. Principles of Tissue Engineering. In Tissue Engineering Made Easy. Edited by Akter, F., 3-16,Academic Press. London, UK (2016).

2. Van Vlierberghe, S., Dubruel, P., Schacht, E. Biopolymer-Based Hydrogels as Scaffolds for Tissue Engineering Applications: A Review. Biomacromolecules. 12 (5), 1387-1408 (2011).

3. Murugan, R., Ramakrishna, S. Nano-featured scaffolds for tissue engineering: A review of spinning methodologies. Tissue Engineering. 12 (3), 435-447 (2006).

4. Hollister, S. J. Porous scaffold design for tissue engineering. Nature Materials. 4 (7), 518-524 (2005).

5. Jun, I., Han, H. S., Edwards, J. R., Jeon, H. Electrospun fibrous scaffolds for tissue engineering: Viewpoints on architecture and fabrication. International Journal of Molecular Sciences. 19 (3), E745 (2018).

6. Rashidi, H. et al. 3D human liver tissue from pluripotent stem cells displays stable phenotype in vitro and supports compromised liver function in vivo. Archives of Toxicology. 92 (10), 3117-3129 (2018).

7. Grant, R., Hay, D. C., Callanan, A. A Drug-Induced Hybrid Electrospun Poly-Capro-Lactone: Cell-Derived Extracellular Matrix Scaffold for Liver Tissue Engineering. Tissue Engineering Part A. 23 (13-14), 650-662 (2017).

8. Munir, N., McDonald, A., Callanan, A. A combinatorial approach: Cryo-printing and electrospinning hybrid scaffolds for cartilage tissue engineering. Bioprinting. 16, e00056 (2019).

9. Burton, T.P., Corcoran, A., Callanan, A. The effect of electrospun polycaprolactone scaffold morphology on human kidney epithelial cells. Biomedical Materials. 13 (1), 015006 (2018).

10. Reid, J. A., Callanan, A. Influence of aorta extracellular matrix in electrospun polycaprolactone scaffolds. Journal of Applied Polymer Science. 136 (44), 48181 (2019).

11. Grant, R., Hallett, J., Forbes, S., Hay, D., Callanan, A. Blended electrospinning with human liver extracellular matrix for engineering new hepatic microenvironments. Scientific Reports. 9 (1), 6293 (2019).

12. Grant, R., Hay, D., Callanan, A. From scaffold to structure: The synthetic production of cell derived extracellular matrix for liver tissue engineering. Biomedical Physics and Engineering Express. 4 (6),065015 (2018).

13. Reid, J. A., Callanan, A. Hybrid cardiovascular sourced extracellular matrix scaffolds as possible platforms for vascular tissue engineering. Journal of Biomedical Materials Research Part B: Applied Biomaterials. 1-15 (2019).

14. Chen, C. S. Mechanotransduction - A field pulling together? Journal of Cell Science. 121 (20), 3285-3292 (2008).

15. Wang, N. Review of cellular mechanotransduction. Journal of Physics D: Applied Physics. 50 (23), 233002 (2017). 
16. Fan, Y. L., Zhao, H. C., Li, B., Zhao, Z. L., Feng, X. Q. Mechanical Roles of F-Actin in the Differentiation of Stem Cells: A Review. ACS Biomaterials Science and Engineering. 5 (8), 3788-3801 (2019).

17. Davidson, A. J., Wood, W. Unravelling the Actin Cytoskeleton: A New Competitive Edge? Trends in Cell Biology. 26 (8), $569-576$ (2016).

18. Blanchoin, L., Boujemaa-Paterski, R., Sykes, C., Plastino, J. Actin dynamics, architecture, and mechanics in cell motility. Physiological Reviews. 94 (1), 235-263 (2014).

19. Guvendiren, M., Burdick, J. A. The control of stem cell morphology and differentiation by hydrogel surface wrinkles. Biomaterials. 31 (25), 6511-6518 (2010)

20. Wan, L. Q. et al. Geometric control of human stem cell morphology and differentiation. Integrative Biology. 2 (7-8), 346 (2010).

21. Park, J. S. et al. The effect of matrix stiffness on the differentiation of mesenchymal stem cells in response to TGF- $\beta$. Biomaterials. 32 (16), 3921-3930 (2011).

22. Evans, N. et al. Substrate stiffness affects early differentiation events in embryonic stem cells. European Cells and Materials. 18, 1-14 (2009).

23. Stukel, J. M., Willits, R. K. Mechanotransduction of Neural Cells Through Cell-Substrate Interactions. Tissue Engineering Part B: Reviews. 22 (3), 173-182 (2016).

24. Charbonier, F. W., Zamani, M., Huang, N. F. Endothelial Cell Mechanotransduction in the Dynamic Vascular Environment. Advanced Biosystems. 3 (2), 1800252 (2019).

25. Nyitray, C. E., Chavez, M. G., Desai, T. A. Compliant 3D Microenvironment Improves $\beta$-Cell Cluster Insulin Expression Through Mechanosensing and $\beta$-Catenin Signaling. Tissue Engineering Part A. 20 (13-14), 1888-1895 (2014).

26. Baker, B. M. et al. Cell-mediated fiber recruitment drives extracellular matrix mechanosensing in engineered fibrillar microenvironments. Nature Materials. 14 (12), 1262-1268 (2015).

27. Evans, N. D., Gentleman, E. The role of material structure and mechanical properties in cell-matrix interactions. Journal of Materials Chemistry B. 2 (17), 2345 (2014).

28. Tusan, C. G. et al. Collective Cell Behavior in Mechanosensing of Substrate Thickness. Biophysical Journal. 114 (11), $2743-2755$ (2018).

29. Hotaling, N. A., Bharti, K., Kriel, H., Simon, C. G. DiameterJ: A validated open source nanofiber diameter measurement tool. Biomaterials. 61, 327-338 (2015).

30. Cox, T. R., Erler, J. T. Remodeling and homeostasis of the extracellular matrix: Implications for fibrotic diseases and cancer. DMM Disease Models and Mechanisms. 4 (2), 165-178 (2011).

31. Haider, A., Haider, S., Kang, I. K. A comprehensive review summarizing the effect of electrospinning parameters and potential applications of nanofibers in biomedical and biotechnology. Arabian Journal of Chemistry. 11 (8), 1165-1188 (2018).

32. De Vrieze, S. et al. The effect of temperature and humidity on electrospinning. Journal of Materials Science. 44 (5), 1357-1362 (2009).

33. Hohman, M. M., Shin, M., Rutledge, G., Brenner, M. P. Electrospinning and electrically forced jets. I. Stability theory. Physics of Fluids. 13 (8), 2201-2220 (2001).

34. Yarin, A. L., Koombhongse, S., Reneker, D. H. Bending instability in electrospinning of nanofibers. Journal of Applied Physics. 89 (5), 3018-3026 (2001).

35. Feltz, K. P., Kalaf, E. A. G., Chen, C., Martin, R. S., Sell, S. A. A review of electrospinning manipulation techniques to direct fiber deposition and maximize pore size. Electrospinning. 1, 46-61 (2017).

36. Simonet, M., Schneider, O. D., Neuenschwander, P., Stark, W. J. Ultraporous 3D polymer meshes by low-temperature electrospinning: Use of ice crystals as a removable void template. Polymer Engineering \& Science. 47 (12), 2020-2026 (2007).

37. Dai, Z., Ronholm, J., Tian, Y., Sethi, B., Cao, X. Sterilization techniques for biodegradable scaffolds in tissue engineering applications. Journal of Tissue Engineering. 7, 204173141664881 (2016).

38. Rogers, W. J. Sterilisation techniques for polymers. In Sterilisation of Biomaterials and Medical Devices. Edited by Lerouge, S., Simmons, A., 151-211, Elsevier Science.Amsterdam, Netherlands (2012).

39. Jokinen, V., Suvanto, P., Franssila, S. Oxygen and nitrogen plasma hydrophilization and hydrophobic recovery of polymers. Biomicrofluidics. 6 (1), 16501-1650110 (2012).

40. Recek, N. et al. Cell Adhesion on Polycaprolactone Modified by Plasma Treatment. International Journal of Polymer Science. 2016, 1-9 (2016).

41. Fleck, N. A., Deshpande, V. S., Ashby, M. F. Micro-architectured materials: Past, present and future. Proceedings of the Royal Society A: Mathematical, Physical and Engineering Sciences. 466 (2121), 2495-2516 (2010).

42. Cooper, A., Bhattarai, N., Zhang, M. Fabrication and cellular compatibility of aligned chitosan-PCL fibers for nerve tissue regeneration. Carbohydrate Polymers. 85 (1), 149-156 (2011).

43. Patel, K. H. et al. Aligned nanofibers of decellularized muscle ECM support myogenic activity in primary satellite cells in vitro. Biomedical Materials. 14 (3), 035010 (2019).

44. Wang, L. et al. Fabrication of open-porous PCL/PLA tissue engineering scaffolds and the relationship of foaming process, morphology, and mechanical behavior. Polymers for Advanced Technologies. 30 (10), 2539-2548 (2019).

45. Sartore, L., Inverardi, N., Pandini, S., Bignotti, F., Chiellini, F. PLA/PCL-based foams as scaffolds for tissue engineering applications. Materials Today: Proceedings. 7, 410-417 (2019).

46. Munir, N., Callanan, A. Novel phase separated polycaprolactone/collagen scaffolds for cartilage tissue engineering. Biomedical Materials (Bristol). 13 (5), 051001 (2018).

47. Wang, W. et al. Fabrication of heterogeneous porous bilayered nanofibrous vascular grafts by two-step phase separation technique. Acta Biomaterialia. 79, 168-181 (2018).

48. Kim, C. et al. Stem Cell Mechanosensation on Gelatin Methacryloyl (GelMA) Stiffness Gradient Hydrogels. Annals of Biomedical Engineering. 48 (2), 893-902 (2019).

49. Chen, Z. et al. 3D Printing of Multifunctional Hydrogels. Advanced Functional Materials. 29 (20), 1900971 (2019).

50. Lee, J. M., Ng, W. L., Yeong, W. Y. Resolution and shape in bioprinting: Strategizing towards complex tissue and organ printing. Applied Physics Reviews. 6,011307 (2019).

51. Zimmermann, R. et al. High resolution bioprinting of multi-component hydrogels. Biofabrication. 11 (4), 045008 (2019). 